\title{
A Generalization of Eneström-Kakeya Theorem and a Zero Free Region of a Polynomial
}

\author{
Mushtaq Ahmad Shah1, Ram Swroop ${ }^{2}$, Humayun Mohd Sofi ${ }^{2}$, Insha Nisar ${ }^{3}$ \\ ${ }^{1}$ G.C.E.T Safapora Ganderbal Kashmir, Jammu and Kashmir, India \\ ${ }^{2}$ NIMS University, Jaipur, India \\ ${ }^{3}$ Mewar University, Rajasthan, India \\ Email: mushtaqa022@gmail.com,mathsram09@gmail.com, sofihemayou0171@gmail.com, inshajan129@gmail.com
}

How to cite this paper: Shah, M.A., Swroop, R., Sofi, H.M. and Nisar, I. (2021) A Generalization of Eneström-Kakeya Theorem and a Zero Free Region of a Polynomial. Journal of Applied Mathematics and Physics, 9, 1271-1277. https://doi.org/10.4236/jamp.2021.96087

Received: May 9, 2021

Accepted: June 15, 2021

Published: June 18, 2021

Copyright ( 2021 by author(s) and Scientific Research Publishing Inc. This work is licensed under the Creative Commons Attribution International License (CC BY 4.0).

http://creativecommons.org/licenses/by/4.0/

\begin{abstract}
For the polynomial $P(z)=\sum_{j=0}^{n} a_{j} z^{j}, \quad a_{j} \geq a_{j-1}, \quad a_{0}>0, \quad j=1,2, \cdots, n$, $a_{n}>0$, a classical result of Eneström-Kakeya says that all the zeros of $P(z)$ lie in $|z| \leq 1$. This result was generalised by A. Joyall and G. Labelle, where they relaxed the non-negativity condition on coefficients. It was further generalized by M.A Shah by relaxing the monotonicity of some coefficients. In this paper, we use some known techniques and provide some more generalizations of the above results by giving more relaxation to the conditions.
\end{abstract}

\section{Keywords}

Polynomial, Zeros, Eneström-Kakeya Theorem

\section{Introduction}

If $P(z)=\sum_{j=0}^{n} a_{j} z^{j}$ is a polynomial of degree $n$. Then Eneström-Kakeya [1] [2] proved the following interesting result.

Theorem A: Let $P(z)=\sum_{j=0}^{n} a_{j} z^{j}$ be a polynomial of degree $n$ such that $a_{n} \geq a_{n-1} \geq \cdots \geq a_{0}>0$, then $P(z)$ has all its zeros in $|z| \leq 1$.

For example: The Polynomial $10 z^{6}+8 z^{5}+7 z^{4}+7 z^{3}+6 z^{2}+2 z+1$ has all zeros in $|z| \leq 1$.

In the literature, there exist several extensions and generalizations of this theorem. Joyal et al. [3] extended Theorem A to the polynomials whose coefficients are monotonic but not necessarily non-negative. In fact, they proved the 
following result.

Theorem B: Let $P(z)=\sum_{j=0}^{n} a_{j} z^{j}$ be a polynomial of degree $n$ such that $a_{n} \geq a_{n-1} \geq \cdots \geq a_{1} \geq a_{0}$, then $P(z)$ has all its zeros in the disk

$$
|z| \leq \frac{1}{\left|a_{n}\right|}\left(\left|a_{n}\right|-a_{0}+\left|a_{0}\right|\right) \text {. }
$$

For example: Consider the Polynomial $4 z^{6}+3 z^{5}+2 z^{4}-z^{2}-z-3$

Here $n=6, a_{n}=4$ and $a_{0}=-3$

Then the zeros of this polynomial lie in

$$
|z| \leq \frac{4-(-3)+3}{4}=\frac{4+3+3}{4}=\frac{10}{4}=\frac{5}{2}
$$

i.e. $|z| \leq 2.5$

The above results were generalised by M.A. Shah [4]. In fact he proved the following result.

Theorem C: Let $P(z)=a_{n} z^{n}+a_{n-1} z^{n-1}+\cdots+a_{p} z^{p}+a_{p-1} z^{p-1}+\cdots+a_{1} z+a_{0}$ be a polynomial of degree $n$ satisfying

$$
a_{p} \geq a_{p-1} \geq \cdots \geq a_{1} \geq a_{0}, p=0,1,2, \cdots, n \text { and } M_{p}=\sum_{j=p+1}^{n}\left|a_{j}-a_{j-1}\right|,
$$

then all the zeros of $P(z)$ lie in the disc

$$
|z| \leq \frac{M_{p}+a_{p}-a_{0}+\left|a_{0}\right|}{a_{n}} .
$$

Theorem D: Let $P(z)=a_{n} z^{n}+a_{n-1} z^{n-1}+\cdots+a_{p} z^{p}+a_{p-1} z^{p-1}+\cdots+a_{1} z+a_{0}$ be a polynomial of degree $n$ satisfying

$$
a_{p} \geq a_{p-1} \geq \cdots \geq a_{1} \geq a_{0}, p=0,1,2, \cdots, n \text { and } M_{p}=\sum_{j=p+1}^{n}\left|a_{j}-a_{j-1}\right|,
$$

then $P(z)$ does not vanish in

$$
|z|<\min \left[1, \frac{\left|a_{0}\right|}{\left|a_{n}\right|+M_{p}+a_{p}-a_{0}}\right] .
$$

In literature [5]-[12], there exist several other generations and extensions of Eneström-Kakeya Theorem. Our main purpose is to relax some conditions on the monotonicity of coefficients and obtain some interesting generalizations of known results.

\section{Main Results}

This paper provides some further generalizations of the Eneström-Kakeya theorem and the above results. In this direction, we first prove the following result.

Theorem 1. Let

$P(z)=a_{n} z^{n}+a_{n-1} z^{n-1}+\cdots+a_{p} z^{p}+a_{p-1} z^{p-1}+\cdots+a_{q} z^{q}+a_{q-1} z^{q-1}+\cdots+a_{1}+a_{0} \quad b e$ a polynomial of degree $n$ satisfying

$$
a_{p} \geq a_{p-1} \geq \cdots \geq a_{q}, p \geq q .
$$




$$
M_{p}=\sum_{j=p+1}^{n}\left|a_{j}-a_{j-1}\right| \text { and } M_{q}=\sum_{j=1}^{q}\left|a_{j}-a_{j-1}\right|,
$$

then all the zeros of $P(z)$ lie in the disk

$$
|z| \leq \frac{M_{p}+M_{q}+a_{p}-a_{q}+\left|a_{0}\right|}{\left|a_{n}\right|} .
$$

Proof. Consider the polynomial

$$
\begin{aligned}
F(z)= & (1-z) P(z) \\
= & (1-z)\left(a_{n} z^{n}+a_{n-1} z^{n-1}+\cdots+a_{p} z^{p}+a_{p-1} z^{p-1}+\cdots\right. \\
& \left.+a_{q} z^{q}+a_{q-1} z^{q-1}+\cdots+a_{1} z+a_{0}\right) \\
= & -a_{n} z^{n+1}+\left[\left(a_{n}-a_{n-1}\right) z^{n}+\left(a_{n-1}-a_{n-2}\right) z^{n-1}+\cdots+\left(a_{p+1}-a_{p}\right) z^{p+1}\right. \\
& +\left(a_{p}-a_{p-1}\right) z^{p}+\left(a_{p-1}-a_{p-2}\right) z^{p-1}+\cdots+\left(a_{q+1}-a_{q}\right) z^{q+1} \\
& \left.+\left(a_{q}-a_{q-1}\right) z^{q}+\left(a_{q-1}-a_{q-2}\right) z^{q-1}+\cdots+\left(a_{1}-a_{0}\right) z+a_{0}\right]
\end{aligned}
$$

This gives

$$
\begin{aligned}
|F(z)| \geq & \left|a_{n}\right||z|^{n+1}-\left[\mid\left(a_{n}-a_{n-1}\right) z^{n}+\left(a_{n-1}-a_{n-2}\right) z^{n-1}+\cdots+\left(a_{p+1}-a_{p}\right) z^{p+1}\right. \\
& +\left(a_{p}-a_{p-1}\right) z^{p}+\left(a_{p-1}-a_{p-2}\right) z^{p-1}+\cdots+\left(a_{q+1}-a_{q}\right) z^{q+1} \\
& \left.+\left(a_{q}-a_{q-1}\right) z^{q}+\left(a_{q-1}-a_{q-2}\right) z^{q-1}+\cdots+\left(a_{1}-a_{0}\right) z+a_{0} \mid\right] \\
\geq & \left|a_{n}\right||z|^{n+1}-\left[\left|a_{n}-a_{n-1}\right||z|^{n}+\left|a_{n-1}-a_{n-2}\right||z|^{n-1}+\cdots+\left|a_{p+1}-a_{p}\right||z|^{p+1}\right. \\
& +\left|a_{p}-a_{p-1}\right||z|^{p}+\left|a_{p-1}-a_{p-2}\right||z|^{p-1}+\cdots+\left|a_{q+1}-a_{q}\right||z|^{q+1} \\
& \left.+\left|a_{q}-a_{q-1}\right||z|^{q}+\left.\left|a_{q-1}-a_{q-2}\right| z\right|^{q-1}+\cdots+\left|a_{1}-a_{0}\right||z|+\left|a_{0}\right|\right] \\
\geq & |z|^{n}\left[\left|a_{n}\right||z|-\left(\left|a_{n}-a_{n-1}\right|+\frac{\left|a_{n-1}-a_{n-2}\right|}{|z|}+\cdots+\frac{\left|a_{p}-a_{p-1}\right|}{|z|^{n-p}}+\cdots\right.\right. \\
& \left.\left.+\frac{a_{q}-a_{q-1}}{|z|^{n-q}}+\cdots+\frac{\left|a_{1}-a_{0}\right|}{|z|^{n-1}}+\frac{\left|a_{0}\right|}{|z|^{n}}\right)\right]
\end{aligned}
$$

Now let $|z|>1$, so that $\frac{1}{|z|^{n-j}}<1,0 \leq j \leq n$, then we have

$$
\begin{aligned}
|F(z)|> & |z|^{n}\left[\left|a_{n}\right||z|-\left(\left|a_{n}-a_{n-1}\right|+\left|a_{n-1}-a_{n-2}\right|+\cdots+\left|a_{p}-a_{p-1}\right|+\cdots\right.\right. \\
& \left.\left.+\left|a_{q}-a_{q-1}\right|+\cdots+\left|a_{1}-a_{0}\right|+\left|a_{0}\right|\right)\right] \\
= & |z|^{n}\left[\left|a_{n}\right||z|-\left(\left|a_{n}-a_{n-1}\right|+\left|a_{n-1}-a_{n-2}\right|+\cdots+\left|a_{p+1}-a_{p}\right|\right.\right. \\
& \left.\left.+a_{p}-a_{p-1}+\cdots+a_{q+1}-a_{q}+\left|a_{q}-a_{q-1}\right|+\cdots+\left|a_{1}-a_{0}\right|+\left|a_{0}\right|\right)\right] \\
= & |z|^{n}\left[\left|a_{n}\right||z|-\left(\sum_{j=p+1}^{n}\left|a_{j}-a_{j-1}\right|+\sum_{j=1}^{q}\left|a_{j}-a_{j-1}\right|+a_{p}-a_{q}+\left|a_{0}\right|\right)\right] \\
= & |z|^{n}\left[\left|a_{n}\right||z|-\left(M_{p}+M_{q}+a_{p}-a_{q}+\left|a_{0}\right|\right)\right. \\
> & 0, \text { if }|z|\left|a_{n}\right|>\left(M_{p}+M_{q}+a_{p}-a_{q}+\left|a_{0}\right|\right)
\end{aligned}
$$


i.e. if

$$
|z|>\frac{M_{p}+M_{q}+a_{p}-a_{q}+\left|a_{0}\right|}{\left|a_{n}\right|}
$$

where $M_{p}=\sum_{j=p+1}^{n}\left|a_{j}-a_{j-1}\right|$ and $M_{q}=\sum_{j=1}^{q}\left|a_{j}-a_{j-1}\right|$.

Thus all the zeros of $F(z)$ whose modulus is greater than 1 lie in the disk

$$
|z| \leq \frac{M_{p}+M_{q}+a_{p}-a_{q}+\left|a_{0}\right|}{\left|a_{n}\right|}
$$

But the zeros of $F(z)$ whose modulus is less than or equal to 1 already satisfy the above inequality and all the zeros of $P(z)$ are also the zeros of $F(z)$. Hence it follows that all the zeros of $P(z)$ lie in the disk

$$
|z| \leq \frac{M_{p}+M_{q}+a_{p}-a_{q}+\left|a_{0}\right|}{\left|a_{n}\right|}
$$

This completes the proof of the Theorem.

For example: Consider the polynomial

$$
10 z^{10}-z^{9}+2 z^{8}-3 z^{7}+4 z^{6}+3 z^{5}+2 z^{4}-z^{3}+3 z^{2}-2 z+1
$$

Here $n=10, a_{n}=10, p=6, q=3, a_{p}=4, a_{q}=-1, a_{0}=1, M_{p}=26$ and $M_{q}=12$

$$
\begin{gathered}
|z| \leq \frac{26+12+4+1+1}{10} \\
|z| \leq 4.4
\end{gathered}
$$

Remark. For $p=n$ and $q=0$, theorem 1 reduces to theorem B.

Applying theorem 1 to the polynomial $p(t z)$, we get the following result Corollary. Let

$P(z)=a_{n} z^{n}+a_{n-1} z^{n-1}+\cdots+a_{p} z^{p}+a_{p-1} z^{p-1}+\cdots+a_{q} z^{q}+a_{q-1} z^{q-1}+\cdots+a_{1}+a_{0}$ be a polynomial of degree $n$ such that for any $t>0$,

$$
t^{p} a_{P} \geq t^{p-1} a_{p-1} \geq \cdots \geq t^{q+1} a_{q+1} \geq t^{q} a_{q}
$$

then all the zeros of $P(z)$ lie in the disk

$$
|z| \leq \sum_{j=p+1}^{n} \frac{\left|t a_{j}-a_{j-1}\right|}{\left|a_{n}\right| t^{n-j+1}}+\sum_{j=1}^{q} \frac{\left|t a_{j}-a_{j-1}\right|}{\left|a_{n}\right| t^{n-j+1}}+\frac{t^{P} a_{p}-t^{q} a_{q}+\left|a_{0}\right|}{t^{n}\left|a_{n}\right|}
$$

Remark. for $q=0$ the above theorem reduces to theorem C.

Next, we prove the following result concerning the zero-free region of a polynomial. In fact we prove the following:

Theorem 2. Let

$P(z)=a_{n} z^{n}+a_{n-1} z^{n-1}+\cdots+a_{p} z^{p}+a_{p-1} z^{p-1}+\cdots+a_{q} z^{q}+a_{q-1} z^{q-1}+\cdots+a_{1}+a_{0} \quad b e$ a polynomial of degree $n$ satisfying

$$
\begin{gathered}
a_{p} \geq a_{p-1} \geq \cdots \geq a_{q}, p \geq q . \\
M_{p}=\sum_{j=p+1}^{n}\left|a_{j}-a_{j-1}\right| \text { and } M_{q}=\sum_{j=1}^{q}\left|a_{j}-a_{j-1}\right|
\end{gathered}
$$


then $P(z)$ does not vanish in

$$
|z|<\min \left[1, \frac{\left|a_{0}\right|}{\left|M_{p}+M_{q}+a_{p}-a_{q}+\right| a_{n}||}\right]
$$

Proof. Consider the reciprocal polynomial

$$
R(z)=z^{n} p\left(\frac{1}{z}\right)=a_{0} z^{n}+a_{1} z^{n-1}+\cdots+a_{q} z^{n-q}+\cdots+a_{p} z^{n-p}+\cdots+a_{n-1} z+a_{n} .
$$

Let

$$
\begin{aligned}
S(z)= & (1-z) R(z) \\
= & (1-z)\left[a_{0} z^{n}+a_{1} z^{n-1}+\cdots+a_{q} z^{n-q}+\cdots+a_{p} z^{n-p}+\cdots+a_{n-1} z+a_{n}\right] \\
= & -a_{0} z^{n+1}+\left(a_{0}-a_{1}\right) z^{n}+\cdots+\left(a_{q+1}-a_{q}\right) z^{n-q}+\cdots+\left(a_{p}-a_{p+1}\right) z^{n-p} \\
& +\cdots+\left(a_{n-1}-a_{n}\right) z+a_{n} .
\end{aligned}
$$

This gives

$$
\begin{aligned}
|S(z)| \geq & \left|a_{0}\right||z|^{n+1}-\left[\left\{\left|a_{0}-a_{1}\right||z|^{n}+\cdots+\left|a_{q+1}-a_{q}\right||z|^{n-q}+\cdots\right.\right. \\
& \left.\left.+\left|a_{p}-a_{p+1}\right||z|^{n-p}+\cdots+\left|a_{n-1}-a_{n}\right||z|+\left|a_{n}\right|\right\}\right] \\
= & |z|^{n}\left[\left|a_{0}\right||z|-\left(\left|a_{0}-a_{1}\right|+\cdots+\frac{a_{q-1}-a_{q}}{|z|^{q-1}}+\frac{\left|a_{q+1}-a_{q}\right|}{|z|^{q}}+\cdots\right.\right. \\
& \left.\left.+\frac{\left|a_{p-1}-a_{p}\right|}{|z|^{p-1}}+\frac{\left|a_{p}-a_{p+1}\right|}{|z|^{p}}+\cdots+\frac{\left|a_{n-1}-a_{n}\right|}{|z|^{n-1}}+\frac{\left|a_{n}\right|}{|z|^{n}}\right)\right] .
\end{aligned}
$$

Now let $|z|>1$, so that $\frac{1}{|z|^{n-j}}<1,0 \leq j \leq n$, then we have

$$
\begin{aligned}
|S(z)| \geq & |z|^{n}\left[\left|a_{0}\right||z|-\left(\left|a_{0}-a_{1}\right|+\cdots+\left|a_{q-1}-a_{q}\right|+\left|a_{q+1}-a_{q}\right|+\cdots\right.\right. \\
& \left.\left.+\left|a_{p-1}-a_{p}\right|+\left|a_{p}-a_{p+1}\right|+\cdots+\left|a_{n-1}-a_{n}\right|+\left|a_{n}\right|\right)\right] \\
= & |z|^{n}\left[\left|a_{0}\right||z|-\sum_{j=p+1}^{n}\left|a_{j}-a_{j-1}\right|+\sum_{j=1}^{q}\left|a_{j}-a_{j-1}\right|+a_{q+1}-a_{q}\right. \\
& \left.+a_{q+2}-a_{q+1}+\cdots+a_{p+1}-a_{p-2}+a_{p}-a_{p-1}+\left|a_{n}\right|\right] \\
= & |z|^{n}\left[\left|a_{0}\right||z|-\left(M_{p}+M_{q}+\left|a_{n}\right|+a_{p}-a_{q}\right)\right] \\
> & 0, \text { if }|z|\left|a_{0}\right|>\left(M_{p}+M_{q}+a_{p}-a_{q}+\left|a_{n}\right|\right)
\end{aligned}
$$

i.e. if

$$
|z|>\frac{M_{p}+M_{q}+a_{p}-a_{q}+\left|a_{n}\right|}{\left|a_{0}\right|}
$$

where $M_{p}=\sum_{j=p+1}^{n}\left|a_{j}-a_{j-1}\right|$, and $M_{q}=\sum_{j=1}^{q}\left|a_{j}-a_{j-1}\right|$. 
Thus all the zeros of $S(z)$ whose modulus is greater than 1 lie in

$$
|z| \leq \frac{M_{p}+M_{q}+a_{p}-a_{q}+\left|a_{n}\right|}{\left|a_{0}\right|}
$$

Hence all the zeros of $S(z)$ and hence of $R(z)$ lie in

$$
|z| \leq \max \left[1, \frac{M_{p}+M_{q}+a_{p}-a_{q}+\left|a_{n}\right|}{\left|a_{0}\right|}\right]
$$

Therefore all the zeros of $P(z)$ lie in

$$
|z| \geq \min \left[1, \frac{\left|a_{0}\right|}{M_{p}+M_{q}+a_{p}-a_{q}+\left|a_{n}\right|}\right]
$$

Thus the polynomial $P(z)$ does not vanish in

$$
|z|<\min \left[1, \frac{\left|a_{0}\right|}{M_{p}+M_{q}+a_{p}-a_{q}+\left|a_{n}\right|}\right]
$$

This completes the proof of the Theorem.

For example: Consider the polynomial $2 z^{8}-5 z^{7}+7 z^{6}+2 z^{5}-2 z^{3}+z^{2}-3 z+10$

Here $n=8, a_{n}=2, p=5, q=3, a_{p}=2, a_{q}=-2, a_{0}=10, M_{p}=24$ and $M_{q}=20$

$$
\begin{gathered}
|z|<\min \left[1, \frac{\left|a_{0}\right|}{\left|M_{p}+M_{q}+a_{p}-a_{q}+\right| a_{n} \mid}\right] \\
\text { i.e., }|z|<\min \left[1, \frac{10}{24+20+2+2+2}\right] \\
\text { i.e., }|z|<\min \left[1, \frac{10}{50}\right] \\
\text { i.e., }|z|<\min (1,0.2) \\
\text { i.e., }|z|<0.2
\end{gathered}
$$

\section{Conclusion and Suggestions}

We can obtain several known results from the above results as special cases. If we apply monotonicity to all the coefficients, we can easily obtain all the previous known results in addition to Eneström-Kakeya theorem.

\section{Acknowledgements}

We send sincere thanks to the members of JAMP for their professional performance and special thanks to managing editor Hellen XU for a rare attitude of high quality.

\section{Conflicts of Interest}

The authors declare no conflicts of interest regarding the publication of this paper. 


\section{References}

[1] Marden, M. (1949) Geometry of Polynomials. Mathematical Surveys and Monographs, Providence, RI. https://doi.org/10.1090/surv/003

[2] Milovanovic, G.V., Mitrinovic, D.S. and Rassias, Th. M. (1994) Topics in Polynomials: Extremal Problems, Inequalities, Zeros. World Scientific Publications, Singapore. https://doi.org/10.1142/1284

[3] Joyal, A., Labelle, G. and Rahman, Q.I. (1967) On the Location of Zeros of Polynomials. Canadian Mathematical Bulletin, 10, 53-63. https://doi.org/10.4153/CMB-1967-006-3

[4] Shah, M.A. (2018) On the Regions Containing Zeros and Zero Free Regions of a Polynomial. International Journal of Advance Research in Science and Engineering, 7, 2761-2772.

[5] Anderson, N., Saff, E.B. and Verga, R.S. (1981) An Extension of Eneström-Kakeya Theorem and Its Sharpness. SIAM Journal on Mathematical Analysis, 12, 10-22. https://doi.org/10.1137/0512002

[6] Aziz, A. and Zargar, B.A. (1996) Some Extensions of Enströme-Kakeya Theorem. Glasnik Matematicki, 31, 239-244.

[7] Aziz, A. and Zargar, B.A. (2012) Bounds for the Zeros of a Polynomial with Restricted coefficients. Applied Mathematics, 3, 30-33. https://doi.org/10.4236/am.2012.31005

[8] Dewan, K.K. and Govil, N.K. (1984) On the Eneström-Kakeya Theorem. Journal of Approximation Theory, 42, 239-244. https://doi.org/10.1016/0021-9045(84)90041-8

[9] K.K. Dewan and M. Bidkham (1993) On the Eneström-Kakeya Theorem. Journal of Mathematical Analysis and Applications, 180, 29-36. https://doi.org/10.1006/jmaa.1993.1379

[10] Gardener, R.B. and Govil, N.K. (1997) Some Generalizations of the on the Eneström-Kakeya Theorem. Acta Mathematica Hungarica, 74, 125-134.

https://doi.org/10.1007/BF02697881

[11] Govil, N.K. and Mctume, G.N. (2002) Some Extensions of on the Eneström-Kakeya Theorem. International Journal of Applied Mathematics, 11, 245-253.

[12] Govil, N.K. and Rahman, Q.I. (1968) On the Enestrom-Kakeya Theorem. Tohoku Mathermatical Journal, 20, 126-136. https://doi.org/10.2748/tmj/1178243172 